\title{
Estudo dos vestígios de peixes dos sítios arqueológicos da área de influência da Usina Hidrelétrica Machadinho, Rio Grande do Sul, Brasil
}

\author{
Cláudio Ricken ${ }^{1} \&$ Luiz Roberto Malabarba ${ }^{2}$
}

\begin{abstract}
${ }^{1}$ Departamento de Ciências Biológicas, Universidade do Extremo Sul Catarinense. Avenida Universitária 1105, Caixa Postal 3167, 88806-000 Criciúma, Santa Catarina, Brasil. E-mail: ricken@ecotono.com.br

2 Departamento de Zoologia, Instituto de Biociências, Universidade Federal do Rio Grande do Sul. Avenida Bento Gonçalves 9500, prédio 43435, 91501-970 Porto Alegre, Rio Grande do Sul, Brasil. E-mail: malabarb@ufrgs.br
\end{abstract}

\begin{abstract}
A study of fish traces taken from the archaeological sites of the areas surrounding the Machadinho Hydroelectric power station, Rio Grande do Sul, Brazil. The analysis of the material obtained in the rescue of archaeological sites in the area surrounding the Hydroelectric Plant of Machadinho, Rio Grande do Sul, revealed that a considerable amount of bones and fish scales incorporated in the alimentary remains. Using an osteological collection as reference, we identified remains of Salminus brasiliensis (Cuvier, 1816) (Characidae), Brycon orbignyanus (Valenciennes, 1849) (Characidae), Pogonopoma obscurum Quevedo \& Reis, 2002 (Loricariidae), Hemiancistrus fuliginosus Cardoso \& Malabarba, 1999, Prochilodus lineatus (Valenciennes, 1836) (Prochilodontidae), Schizodon sp. (Anostomidae), Leporinus sp. (Anostomidae), Hoplias sp. (Erythrinidae), Hypostomus sp. (Loricariidae) and Crenicichla sp. (Cichlidae). Based on specimens from fish collections, regressions were obtained comparing the size of the pre-maxillary bone in Crenicichla spp. and the length of the pectoral fin spine of Hemiancistrus fuliginosus, Pogonopoma obscurum and Hypostomus spp, with the standard length and weight of corresponding specimens. The estimated size and weight of the specimens obtained from fish remains in the archaeological sites varied between 79.7-153.9 mm and 13.5-33.9 g for Hemiancistrus fuliginosus, 158.2-151.0 mm and 179.5-194.3 g for Pogonopoma obscurum, 117.1-166.2 mm and 86.6-93.1 g for Crenicichla spp., and 62.2-397.2 mm and 34-20.3 $\mathrm{g}$ for Hypostomus spp. Therefore using these estimates of standard lengths and weights it was possible to formulate a hypotheses on the fishing technology used by the inhabitants of these sites.
\end{abstract}

KEY WORDS. Alimentary remains; archaeology; ichthology; paleoenviroment; zooarcheology.

A importância da análise de restos de peixes em sítios arqueológicos reside na possibilidade de interpretação de diversos contextos ligados a uma época passada, tais como tecnologia de pesca, sazonalidade da exploração e condições paleoambientais do corpo d'água (RENFREW \& BAHN 1991).

Estudos osteológicos de peixes marinhos com detalhamento suficiente para estudos de peças desarticuladas abundam principalmente nas décadas de 50, 60 e 70. No entanto, nem sempre as peças descritas são representadas nos sítios e nem descrevem a grande variedade de espécies presentes na costa atlântica. Para peixes de água doce, existem alguns trabalhos bastante completos de descrição osteológica, mas em geral apresentando desenhos de peças associadas, dificultando a análise e reconhecimento de peças desarticuladas, sendo em sua maioria pouco úteis em estudos arqueológicos. Dentre os trabalhos mais relevantes na identificação de peças se encontram os de Cockerell (1914), Weitzman (1962), Roberts (1969, 1973, 1974), Machado-Allison (1985), Schaefer (1987), Reis \& Malabarba (1988), Vari (1983). Os únicos voltados para descrição de peças diagnósticas desarticuladas são BIsBAL \& GoMEZ
(1986) e Falabella et al. (1995). No primeiro, os autores descreveram detalhadamente a morfologia do espinho peitoral de oito espécies de siluriformes do rio Prata na província de Buenos Aires. No segundo, foram descritas peças cranianas de 33 espécies de peixes do litoral do Chile central.

$\mathrm{Na}$ região Neotropical, destacam-se como manuais direcionados para a identificação de peixes em sítios arqueológicos os trabalhos de Garcia (1970), AbILHôA \& CorrêA (1993) e CorrêA \& Vianna (1992), que restringem-se apenas a espécies marinhas, utilizando otólitos como base para identificação. Otólitos, porém, nem sempre estão presentes nos sítios, principalmente nas camadas mais inferiores. Como conseqüência, existe ainda uma grande dificuldade no resgate, identificação e quantificação de restos destes animais.

Com relação à presença de restos de peixes em sítios arqueológicos do interior, Prous (1992) comenta que os indícios encontrados nos sítios tupi-guarani reforçam a impressão de que a pesca tinha uma importância bem maior que a caça. No entanto apenas muito recentemente os autores têm dado o devido valor à análise deste tipo de material. 
Rosa (2000) publicou o resultado parcial da análise de sítios arqueológicos da região de Corumbá, Mato Grosso do Sul, Brasil. Trata-se do primeiro trabalho em sítios de interior que contempla os peixes como um componente cultural a ser estudado. Apesar disso, o autor do trabalho fornece somente o número mínimo de indivíduos (NMI) das espécies citadas, além de classificar grande parte dos Siluriformes que compõem a maioria dos restos de peixe somente ao nível de família.

Este trabalho procura descrever a composição ictiofaunística dos sítios da área de abrangência da Usina Hidrelétrica Machadinho e avaliar a efetividade de curvas de regressão obtidas a partir de medidas realizadas em determinadas peças para a estimativa do comprimento padrão e peso total dos espécimes encontrados nos sítios arqueológicos. Também teorizamos a respeito de métodos de pesca a partir de dados da composição e dimensões da arqueofauna, assim como, discutimos a composição da ictiofauna encontrada nos sítios em relação à composição atual de espécies do alto rio Uruguai.

\section{MATERIAL E MÉTODOS}

Dos 58 sítios arqueológicos pesquisados pela equipe do Centro de Estudos e Pesquisas Arqueológicas, MCT/PUCRS (CEPA) sete possuíam material faunístico. São os sítios: U 71, U 377, U 391, U 454, U 467, U 458 e U 470 indicados na figura 1. A localização das aldeias segue o padrão descrito por Prous (1992): os sítios ocupam a parte superior da encosta de morros que dominam um rio principal navegável. As fichas de registro com todos os dados descritivos encontram-se nos arquivos do Instituto de Patrimônio Histórico e Arqueológico Nacional (IPHAN).

A lista de espécies utilizada para análise e montagem da coleção osteológica foi baseada em BERTOLETTI et al. (1989a, b) e atualizada com base em Rers et al. (2003). Foi obtido um total de 2907 peças procedentes dos sítios U36, U457, U438 e U 454. O método de separação foi por peneiramento a úmido, em peneira de crivo $3 \mathrm{~mm}$.

Os espécimes recentes ou as peças diagnósticas preparadas para a coleção osteológica de referência pertencem à coleção do Museu de Ciências e Tecnologia Pontifícia Universidade Católica do Rio Grande do Sul (MCP) ou foram capturadas ou adquiridas no comércio para este fim e catalogadas na coleção do Departamento de Zoologia Universidade Federal do Rio Grande do Sul (UFRGS). Para a limpeza, o material foi fervido em uma solução de água e detergente e, posteriormente, teve suas partes moles retiradas para a separação do esqueleto. As peças foram então deixadas imersas em solução digestiva de tripsina tamponada com solução de borato de sódio, conforme método descrito em Cailliet et al. (1986), até a completa remoção dos tecidos. Posteriormente, as peças foram secas ao ar e acondicionadas em frascos plásticos etiquetados. Os espécimes recentes utilizados para a identificação das peças arqueológicas constam nos Apêndices 1 e 2 .
A nomenclatura das peças e denominação das estruturas segue Weitzman (1962) e Castro \& Castro (1987) para Characiformes, Reis \& Malabarba (1988) para Perciformes e SCHAEFer (1987) para os Siluriformes.

Para a estimativa de abundância taxonômica foi utilizado o número de espécimes ósseos identificados (NISP) (GraYson 1984).

Foi procedida a medição, com paquímetro digital com precisão de $0,01 \mathrm{~mm}$, de algumas das estruturas ósseas provenientes do material coletado nos sítios arqueológicos. As estruturas selecionadas para medição foram espinho peitoral em P. obscurum, H. fuliginosus e nas espécies de Hypostomus Lacépède, 1803, medindo-se largura do espinho peitoral (LEP) em sua base no início da ornamentação com odontódios (Fig. 2); e o pré-maxilar nas espécies de Crenicichla Heckel, 1840, medindo-se o comprimento do processo lateral (CPL) do osso pré-maxilar de sua parte mais anterior até a mais posterior (Fig. 3). Estas mesmas medidas e os dados de comprimento padrão (CP) e peso total (PT) foram tomados em exemplares das mesmas espécies ou gêneros provenientes de coleções ictiológicas, para a estimativa de tamanho e peso dos espécimes utilizados pelas populações indígenas. Estas estimativas foram feitas através do cálculo das curvas de regressão linear para as relações LEP (mm) $\mathrm{x}$ CP $(\mathrm{mm})$ e CPL $(\mathrm{mm}) \mathrm{x}$ CP $(\mathrm{mm})$ e exponenciais para LEP $(\mathrm{mm})$ x PT $(\mathrm{g})$ e CPL $(\mathrm{mm})$ x PT $(\mathrm{g})$. Exemplares medidos são listados no Apêndice 3.

\section{RESULTADOS}

Foram identificadas 2050 peças pertencentes a, pelo menos, onze gêneros e sete famílias. As tabelas I e II apresentam o número de espécimes identificados (NISP) obtido para espécimes identificados ao nível de família, gênero ou espécie, acompanhado da identificação de qual peça foi usada para sua determinação.

As peças que demonstraram diagnose mais acurada estão ilustradas nas figuras 4-13 e 14-23. O NISP apresentou um grande número de peças de loricariídeos (Tabs I e II) devido principalmente à ocorrência de placas dérmicas nos sítios.

O número mais alto de peças ósseas foi observado para loricariideos não identificados e Hypostomus spp., demonstrando uma exploração mais intensa deste recurso ou a preservação diferenciada de suas peças. Em terceiro lugar, segue Crenicichla spp. Foi observada a presença elevada de representantes de Pimelodidae identificados através do cleitro, mas observou-se a presença de poucos espinhos peitorais, sendo identificados apenas espinhos de dois exemplares de Rhamdia sp. Foi identificado também um opérculo de B. orbignyanus praticamente extinto atualmente nesta área do rio Uruguai.

As curvas de regressão resultaram todas lineares para as relações LEP $(\mathrm{mm})$ x CP $(\mathrm{mm})$ e CPL $(\mathrm{mm})$ x CP $(\mathrm{mm})$ e exponenciais para LEP (mm) x PT (g) e CPL (mm) x PT (g) (Tab. III). 

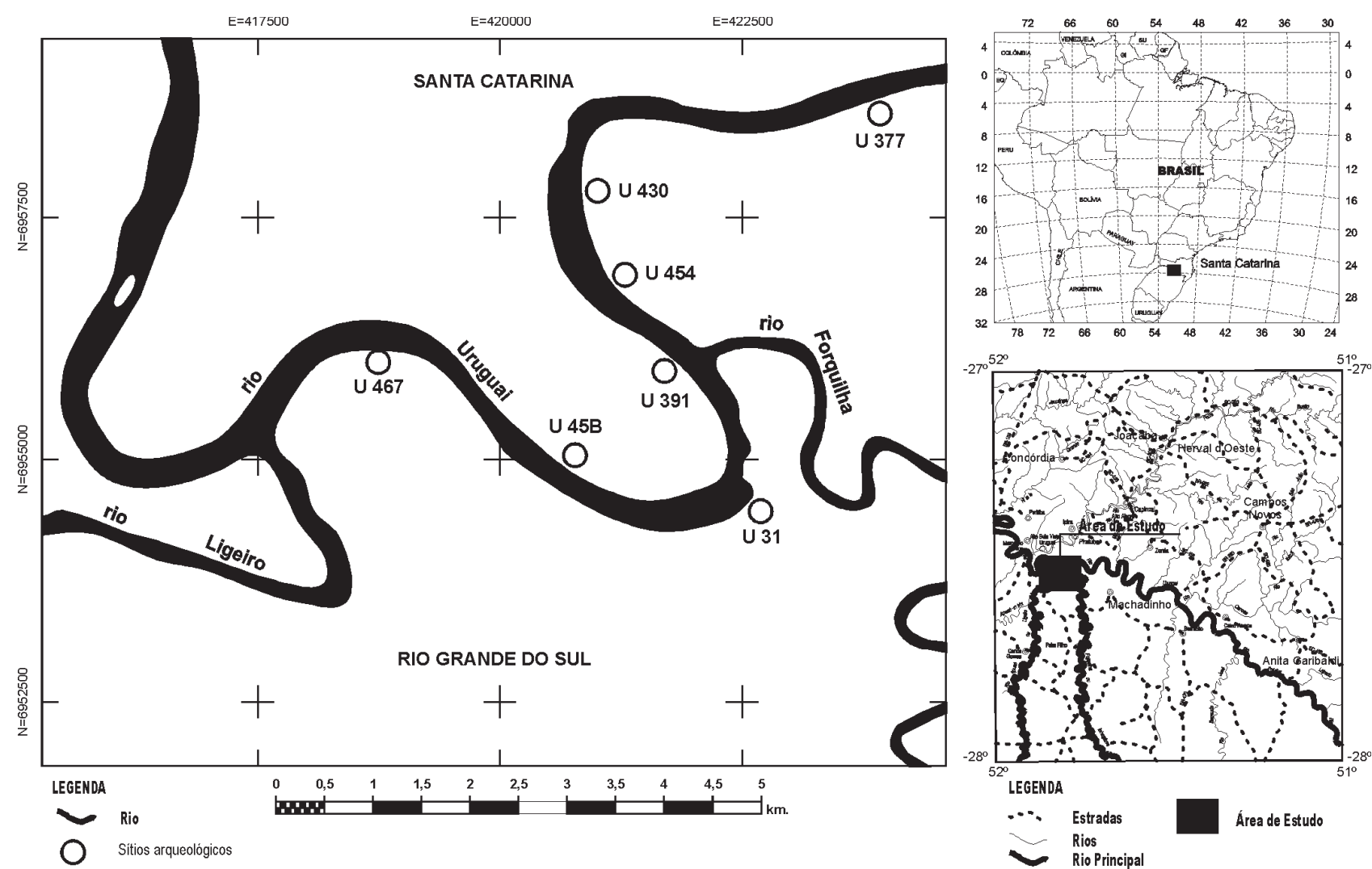

Figura 1. Trecho do Rio Uruguai na área da UHE Machadinho com a localização dos sítios arqueológicos onde foi encontrado material faunístico.

\section{DISCUSSÃO}

A análise dos restos de peixes dos sítios da UHE Machadinho demonstrou que os ossos ângulo-articular, cleitro, dentário, espinho peitoral, hiomandibular, mesetmóide, opérculo, pré-maxilar, pré-opérculo e quadrado foram os mais constantes. Estes apresentaram maior possibilidade de serem diagnosticados até nível de gênero ou espécie, semelhante ao observado por Falabella et al. (1995) e por Needs-Howarth (1999). O mesetmóide, não citado pelos autores, demonstrou ser uma peça bastante presente nos sítios e com potencial para diagnose, sendo a única peça craniana em Loricariidae a apresentar preservação satisfatória para ser utilizada na sua identificação. O maxilar, constantemente citado na literatura, não foi encontrado em nenhum dos sítios estudados.

Owen \& MerRick (1994) postulam não ser apropriada a utilização de dados de grupos integrados de vertebrados para determinar a mudança de tamanho de representantes de um só grupo. No entanto, no caso das regressões do CPL em relação ao CP em Crenicichla spp. e da LEP em relação ao CP em Hypostomus spp., observou-se uma relação estatisticamente significativa entre as duas variáveis mesmo calculando as regressões a partir de amostras compostas de diferentes espécies de Crenicichla e de diferentes espécies de Hypostomus. Desta forma, foi possível estimar o CP de cada espécime destes gêneros encontrados nos sítios mesmo sem a identificação ao nível de espécie dos espinhos peitorais de Hypostomus e dos pré-maxilares de Crenicichla.

As regressões para estimativa do peso dos espécimes demostraram-se estatisticamente significativas para P. obscurum, Hypostomus spp. e Crenicichla spp., mas devem ser analisadas com cautela. De acordo com Owen \& Merrick (1994), dentre outros, estimativas de tamanho dos peixes a partir de ossos desarticulados devem ser relacionadas com medidas realizadas nos ossos; e qualquer consideração subseqüente de peso deve ser enfatizada com os limites de confiança e variações de peso. Isto porque o peso dos espécimes pode variar de acordo com a estação do ano, ciclo reprodutivo e condições de disponibilidade alimentar, entre outros fatores. Este fato foi claramente percebido quando nos confrontamos com a regressão da LEP em relação ao PT, na espécie de $H$. fuliginosus, que apresenta uma grande variabilidade e coeficiente de correlação bastante reduzido $(r=0,632$, Tab. III).

Os aparelhos de pesca são seletivos para tipos particulares e determinados padrões de tamanho. Esta seletividade é refletida 


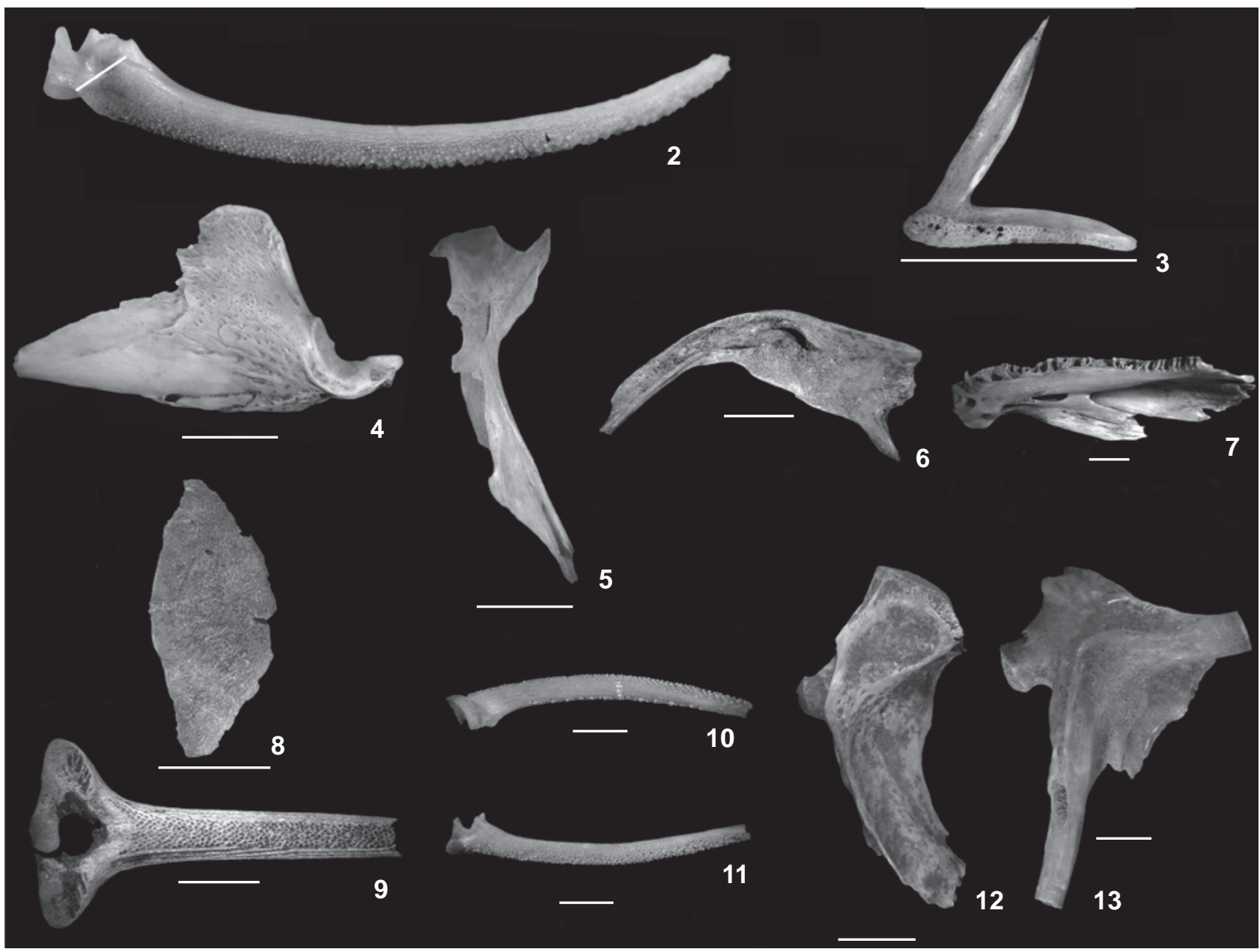

Figuras 2-13. (2) Face ventral do espinho peitoral direito de Hypostomus isbrueckeri demonstrando a medida tomada da largura do espinho peitoral (LEP) em sua base, no início da ornamentação com odontódios. Comprimento ântero-posterior da peça = 7,50 cm. $(3)$ Pré-maxilar esquerdo de Crenicichla vittata vista interna. A medida tomada do comprimento do processo lateral do pré-maxilar (CPL) em Crenicichla spp. é representada pela barra clara. Comprimento ântero-posterior da peça $=1,67 \mathrm{~cm}$. (4-13) Peças identificadas, as escalas representam $5 \mathrm{~mm}$ : (4) ângulo-articular esquerdo de H. malabaricus, vista externa, U 454-A39; (5) cleitro esquerdo de Crenicichla sp., vista externa, U454-A161; (6) cleitro esquerdo de Pimelodidae, vista dorsal, U 470-A383; (7) dentário direito de Hoplias sp., vista interna, U 454-A87; (8) escama ciclóide, vista externa, U454-A165; (9) espinho dorsal de Loricariidae, vista anterior, U 470-A329; (10) espinho peitoral esquerdo de $P$. obscurum, vista ventral, U 454-A90; (11) espinho peitoral direito de $H$. fuliginosus, vista ventral, U454A120; (12) hiomandibular esquerdo de P. lineatus, vista externa, U470-A424; (13) hiomandibular esquerdo de Crenicichla sp., vista externa, U470-A424.

na estrutura populacional dos vestígios de um sítio arqueológico (Greenspan 1998). Os anzóis encontrados no sítio U 454 comprovam o uso deste aparelho. Dentre as espécies registradas que são comumente capturadas em anzóis estão B. orbignyanus, Hoplias sp. S. maxillosus, Crenicichla sp. e Rhamdia sp.

A observação do NMI das espécies identificadas nos demonstra claramente a presença de espécies de pelo menos 11 gêneros nos sítios, porém com a predominância de Hypostomus spp. Uma vez que estes não permitem a captura com anzóis, podemos afirmar que outro tipo de aparelho de pesca era também utilizado nos sítos estudados. Os espécimes de loricariideos encontrados nos sítios apresentam grande variação de tamanho principalmente entre espécimes de Hypostomus. Este fato pode ser interpretado como um tipo de pesca intensiva, conforme sugere Noelli et al. (1995), como armadilhas tipo pari, covo, puçá ou ictiotóxicos. Considerando que as espécies em questão não são susceptíveis a mortandades periódicas em massa, como acontece com algumas espécies de

ZOOLOGIA 26 (3): 469-478, September, 2009 


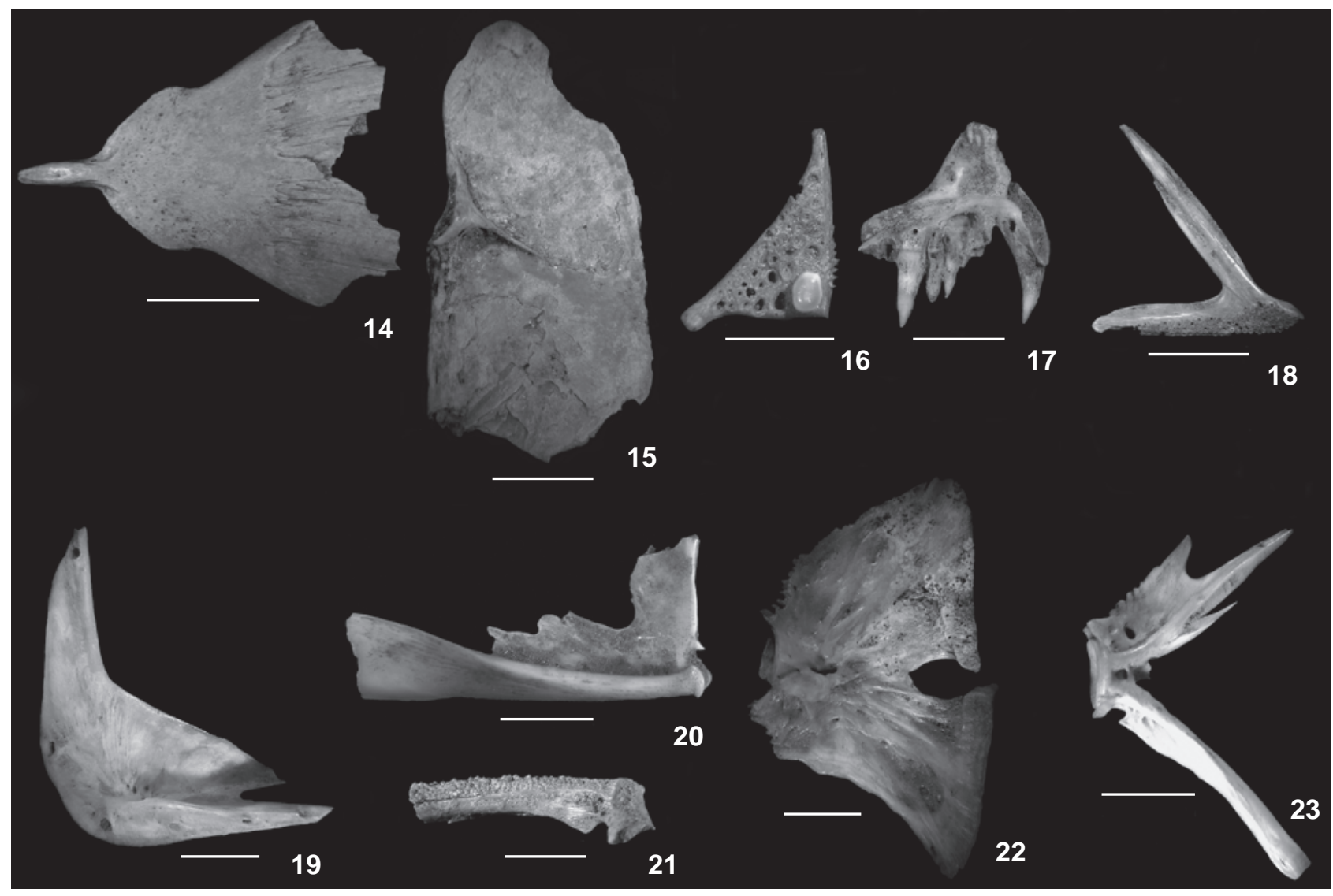

Figuras 14-23. Peças identificadas. As escalas representam 5 mm. (14) mesetmóide de Schizodon sp., vista dorsal, U454-A157; (15) opérculo direito de $B$. orbignyanus, vista interna, U454-A180; (16) placa faringeana de Cichlidae, U377 A-267; (17) pré-maxilar esquerdo de Hoplias sp., vista interna, U454-A6; (18) pré-maxilar esquerdo de Crenicichla sp., vista interna, U454-A139 (19) pré-opérculo direito de Leporinus sp., vista interna, U454-A64; (20) quadrado esquerdo de Leporinus sp., vista interna, U470-A426; (21) raio da nadadeira de Loricariidae, U454-A4; (22) vértebra caudal de Loricariidae, vista lateral, U470-A410; (23) vértebra não identificada, vista lateral, U454-A62.

peixes da América do Norte e Europa, e nem a localização dos sítios favoreceria a deposição, não há dúvidas quanto à origem cultural dos depósitos ocorridos na área da Usina Hidrelétrica Machadinho.

No entanto, se utilizarmos como modelo os levantamentos da ictiofauna realizados por BeRTOLETTI et al. (1989a, b) para o alto rio Uruguai, perceberemos a falta de registro de Characiformes nos depósitos Guaranis. Estes seriam coletados teoricamente em grande quantidade, se utilizássemos um dos métodos de captura em massa citados por Noelli et. al. (1995). Podemos supor que o tipo de processamento dos peixes tenha sido diferenciado. NoeluI et. al. (1995) cita o preparo de farinha (pirá cuí) a partir de pequenos peixes, chamados pelos Guarani como pyquira. Logicamente este procedimento não deixaria restos destes peixes. Porém outras interpretações tafonômicas podem ser dadas, como por exemplo, a não preservação ou a não recuperação das peças procedentes dos espécimes de Characiformes.
Outra ocorrência incomum é o alto número de cleitros de Pimelodidae e o baixo número de espinhos deste grupo, apenas dois identificados como Rhamdia sp. Isto sugere a remoção destes espinhos do local de deposição, seja para o uso como instrumentos ou para outra finalidade.

\section{AGRADECIMENTOS}

A CAPES pela bolsa concedida. A Clarice B. Fialho e Marco A. Azevedo pelo auxílio na análise estatística e Lúcia Safi pela preparação das imagens. Aos arqueólogos Júnior M. Domiks, Gisleni Monticelli e Klaus Hilbert e biólogos José S. Pezzi da Silva e Vinícius A. Bertaco do Museu de Ciências Tecnologia (PUCRS) e Evoy Zanibonni Filho e Samira Meurer do Laboratório de Aqüicultura da UFSC por ceder material de estudo e informações relacionadas. Aos arqueólogos André Luiz Jacobus, Adriana Schmidt Dias e Rodrigo Lavina por oportunizarem participação de trabalhos de campo e sugestões para finalização deste trabalho. 
Tabela I. Listagem número de espécimes identificados a partir das peças ósseas de peixes recuperadas nos sítios da UHE Machadinho. (NI) Não identificado.

\begin{tabular}{|c|c|c|c|c|c|c|c|c|c|}
\hline \multirow{2}{*}{ Peças recuperadas } & \multirow{2}{*}{$\mathrm{NI}$} & \multicolumn{4}{|c|}{ Loricariidae } & \multicolumn{2}{|c|}{ Characidae } & \multicolumn{2}{|c|}{ Anostomidae } \\
\hline & & $\mathrm{NI}$ & Hypostomus sp. & P. obscurum & H. fuliginosus & B. orbignyanus & S. brasileinsis & Leporinus sp. & Schizodon sp. \\
\hline Ângulo-articular & - & - & - & - & - & - & 2 & - & - \\
\hline Basiociptal & - & 1 & - & - & - & - & - & - & - \\
\hline Cintura pélvica & - & 20 & - & - & - & - & - & - & - \\
\hline Cleitro & - & 57 & - & - & - & - & - & 3 & - \\
\hline Coracóide & - & 1 & - & - & - & - & - & - & - \\
\hline Dentário & - & - & - & - & - & - & 1 & - & - \\
\hline Escamas ciclóides & 9 & - & - & - & - & - & - & - & - \\
\hline Escamas ctenóides & 51 & - & - & - & - & - & - & - & - \\
\hline Espinho dorsal & 4 & 5 & - & - & - & - & - & - & - \\
\hline Espinho peitoral & - & 26 & 33 & 6 & 8 & - & - & - & - \\
\hline Fragmentos & 662 & 13 & - & - & - & - & - & - & - \\
\hline Hiomandibular & - & - & - & - & - & - & - & - & - \\
\hline Maxilar & - & - & - & - & - & - & - & - & - \\
\hline Mesetmóide & - & 11 & - & - & - & - & - & - & 2 \\
\hline Opérculo & - & - & - & - & - & 1 & - & 2 & 1 \\
\hline Paresfenóide & 3 & 1 & - & - & - & - & - & - & - \\
\hline Placas dérmicas & - & 1479 & - & - & - & - & - & - & - \\
\hline Placas faringeanas & - & - & - & - & - & - & - & - & - \\
\hline Pós temporal & - & 44 & - & - & - & - & - & - & - \\
\hline Pré-maxilar & - & - & - & - & - & - & - & - & - \\
\hline Pré-opérculo & - & - & - & - & - & - & - & 4 & - \\
\hline Pterigióforo & 6 & 13 & - & - & - & - & - & - & - \\
\hline Quadrado & - & - & - & - & - & - & - & 6 & - \\
\hline Raios nadadeiras & 7 & 42 & - & - & - & - & - & - & - \\
\hline Vértebras & 125 & 189 & - & - & - & - & - & - & - \\
\hline Total & 867 & 1911 & 33 & 6 & 8 & 1 & 3 & 15 & 3 \\
\hline
\end{tabular}

\section{LITERATURA CITADA}

AbILHôA, V. \& M.F.M. CorrêA. 1993. Catálogo de otólitos de Carangidae (Osteichthyes-Perciformes) do litoral do estado do Paraná, Brasil. Nerítica 7: 119-131.

Bertoletti, J.J.; C.A.S. Lucena; Z.M.S. Lucena; L.R. Malabarba \& R.E. ReIs. 1989a. Ictiofauna do rio Uruguai superior entre os municípios de Aratiba e Esmeralda, Rio Grande do Sul, Brasil. Comunicações do Museu de Ciências e Tecnologia da PUCRS 9: 3-42.

Bertoletti, J.J.; C.A.S. Lucena; Z.M.S. Lucena; L.R. Malabarba \& R.E. ReIs. 1989b. Ictiofauna do rio Canoas, sistema do rio Uruguai superior, Campos Novos, Santa Catarina, Brasil.
Comunicações do Museu de Ciências e Tecnologia da PUCRS 49: 43-75.

Bisbal, G.A. \& S.E. Gomez. 1986. Morfologia comparada de la espina pectoral de algunos siluriformes Bonaerenses (Argentina). Physis 44 (107): 81:93.

Cailliet, G.M; M.S. Love \& A.W. Ebeling. 1986. Fishes: a field and laboratory manual on their struture, identification, and natural history. Belmont, Wadsworth, 194p.

Castro R.M.C. \& M.M.C. Castro. 1987. Proposta de uma nomenclatura osteológica para Characiformes (Pisces: Ostariophysi). Boletim do Museu Emílio Goeldi, Série Zoologia, 3 (1): 25-32.

Cockerell, T.D.A. 1914. The scales of the South American 
Tabela II. Listagem número de espécimes identificados a partir das peças ósseas de peixes recuperadas nos sítios da UHE Machadinho. (NI) Não identificado.

\begin{tabular}{|c|c|c|c|c|c|c|c|}
\hline \multirow{2}{*}{ Peças recuperadas } & \multirow{2}{*}{$\mathrm{NI}$} & \multirow{2}{*}{$\frac{\text { Prochilodontidae }}{\text { P. lineatus }}$} & \multirow{2}{*}{$\frac{\text { Erythrinidae }}{\text { Hoplias sp. }}$} & \multicolumn{2}{|r|}{ Cichlidae } & \multicolumn{2}{|c|}{ Pimelodidae } \\
\hline & & & & $\mathrm{NI}$ & Crenicichla spp. & $\mathrm{NI}$ & Rhamdia sp. \\
\hline Ângulo-articular & - & 1 & 3 & - & 1 & - & - \\
\hline Basiociptal & - & - & - & - & - & - & - \\
\hline Cintura pélvica & - & - & - & - & - & - & - \\
\hline Cleitro & - & 1 & 3 & 4 & 7 & 13 & - \\
\hline Coracóide & - & - & - & - & - & - & - \\
\hline Dentário & - & - & - & - & - & - & - \\
\hline Escamas ciclóides & 9 & - & - & - & - & - & - \\
\hline Escamas ctenóides & 51 & - & - & - & - & - & - \\
\hline Espinho dorsal & 4 & - & - & - & - & - & - \\
\hline Espinho peitoral & - & - & - & - & - & 1 & 2 \\
\hline Fragmentos & 662 & - & - & - & - & - & - \\
\hline Hiomandibular & - & 1 & - & - & 1 & - & - \\
\hline Maxilar & - & - & - & - & - & - & - \\
\hline Mesetmóide & - & - & - & - & - & 1 & - \\
\hline Opérculo & - & - & 1 & - & 2 & 3 & - \\
\hline Paresfenóide & 3 & - & - & - & - & - & - \\
\hline Placas dérmicas & - & - & - & - & - & - & - \\
\hline Placas faringeanas & - & - & - & 5 & - & - & - \\
\hline Pós temporal & - & - & - & - & - & - & - \\
\hline Pré-maxilar & - & - & 1 & - & 16 & - & - \\
\hline Pré-opérculo & - & - & 1 & - & 1 & - & - \\
\hline Pterigióforo & 6 & - & - & - & - & - & - \\
\hline Quadrado & - & - & - & - & 1 & - & - \\
\hline Raios nadadeiras & 7 & - & - & - & - & - & - \\
\hline Vértebras & 125 & - & - & - & - & - & - \\
\hline Total & 867 & 3 & 9 & 9 & 29 & 18 & 2 \\
\hline
\end{tabular}

characinid fishes. Annals of the Carnegie Museum 3: 92-113.

CorrêA, M.F. \& M.S. Vianna. 1992. Catálogos de otólitos de Scianidae (Osteichthyes-Perciformes) do litoral do estado do Paraná. Nerítica 7 (1-2): 13-40.

Falabella, F.; R. Melendez. \& M.L. Vargas. 1995. Claves osteológicas para peces de Chile central. Un enfoque arqueológico. Santiago de Chile, Artegrana, 208p.

GARCIA, C.R. 1970. Levantamento ictiológico em jazidas préhistóricas, p. 475 - 486. In: P. Duarte (Ed.). Estudos de PréHistória Geral e Brasileira. São Paulo, Instituto de PréHistória, Universidade de São Paulo, 590p.

Grayson, D. K. 1984. Quantitative zooarchaeology. Florida, Academic Press, 202p.
GreEnSPAN, R.L. 1998. Gear selectivity models, mortality profiles and the interpretation of archaeological fish remains: a case study from Harney Basin, Oregon. Journal of Archaeological Science 25: 973:984

Machado-Allison, A. 1985. Estudios sobre la subfamilia Serrasalminae. Parte III: sobre el status genérico y relaciones filogenéticas de los géneros Pygopristis, Pygocentrus, Pristobrycon y Serrasalmus (Teleostei-Characidae-Serrasalminae). Acta Biologica Venezuelica 12 (1): 19-42.

NeEds-Howarth, S.J. 1999. Native fishing in the Great Lakes - A multidisciplinary approach to zooarchaeological remains from precontact iroquian villages near Lake Simcoe, Ontario. Toronto, Rijksuniversiteit Groningen, 151p. 
Tabela II. Regressões linear e exponencial obtidas a partir das medidas de largura do espinho peitoral (LEP) e comprimento padrão (CP) de $P$. obscurum, $H$. fuliginosus e Hypostomus spp.; e do comprimento do processo lateral do pré-maxilar (CPL) e CP em Crenicichla spp.

\begin{tabular}{|c|c|c|c|c|c|}
\hline \multirow{2}{*}{ Espécies analisadas } & \multirow{2}{*}{ Amostra (N) } & \multicolumn{2}{|c|}{ Regressão linear } & \multicolumn{2}{|c|}{ Regressão exponencial } \\
\hline & & Fórmula resultante & $\mathrm{R}$ & Fórmula resultante & $\mathrm{R}$ \\
\hline \multicolumn{6}{|l|}{ LEP $\times$ CP } \\
\hline P. obscurum & 35 & $y=17,908 x+102,690$ & 0,899 & $y=104,858(0,199 x)$ & 0,789 \\
\hline \multicolumn{6}{|l|}{ LEP $\times C P$} \\
\hline H. fuliginosus & 46 & $y=20,604+30,690$ & 0,978 & $y=23,57(0,24 x)$ & 0,632 \\
\hline \multicolumn{6}{|l|}{ LEP $\times$ CP } \\
\hline H. roseopunctatus & 19 & $y=34,903 x+14,606$ & 0,908 & $y=13,208(0,426 x)$ & 0,908 \\
\hline H. uruguayensis & 15 & & & & \\
\hline H. regani & 22 & & & & \\
\hline H. luteus & 19 & & & & \\
\hline H. isbrueckeri & 37 & & & & \\
\hline H. aspilogaster & 27 & & & & \\
\hline H. commersoni & 26 & & & & \\
\hline \multicolumn{6}{|l|}{$C P L \times C P$} \\
\hline C. vittata & 16 & $y=104,858 x+39,689$ & 0,935 & $y=15,098(0,089 x)$ & 0,881 \\
\hline C. prenda & 6 & & & & \\
\hline C. missioneira & 17 & & & & \\
\hline C. minuano & 20 & & & & \\
\hline C. lepidota & 21 & & & & \\
\hline C. jurubi & 8 & & & & \\
\hline C. gaucho & 14 & & & & \\
\hline C. celidochilus & 15 & & & & \\
\hline C. igara & 13 & & & & \\
\hline C. tendybaguassu & 10 & & & & \\
\hline
\end{tabular}

Noelli, F.S.; L.T. Mota; \& F.A. Silva. 1995. Pari: armadilhas de pesca no sul do Brasil e a arqueologia. Coleção Arqueologia EDIPUCRS 1 (2): 435-446.

Owen, J.F. \& J.R. MerRick. 1994. Analysis of coastal middens in South-Eastern Australia: sizing of fish remains in holocene deposits. Journal of Archaeological Science 21: 3-10.

Prous, A. 1992. Arqueologia Brasileira. Brasília, Editora da Universidade de Brasília, 613p.

Reis, R.E. \& L.R. Malabarba. 1988. Revision of the neotropical cichlid genus Gymnogeophagus Ribeiro, 1918, with descriptions of two new species (Pisces, Perciformes). Revista Brasileira de Zoologia 4 (4): 259-305.

Reis, R.E.; S.O Kullander \& C.J. Ferraris Jr. 2003. Check list of the freshwater fishes of South and Central America. Porto Alegre, EDIPUCRS, 742p.

Renfrew, C. \& P. Bahn. 1991. Arqueología: teorias, métodos y práctica. Madrid, Aklal, 571p.
RoberTs, T. 1969. Osteology and relationships of Characoid fishes, particularly the genera Hepsetus, Salminus, Hoplias, Ctenolucius, and Acestrorhynchus. Proceedings of the California Academy of Sciences 36 (15): 391-500.

Roberts, T. 1973. Osteology and relationships of the Prochilodontidae, a south american family of Characoid fishes. Bulletin of the Museum of Comparative Zoology 145 (4): 213-239.

RoBERTS, T. 1974. Osteology and classification of the neotropical Characoid fishes of the families Hemiodontidae (Including Anodontinae) and Parodontidae. Bulletin of the Museum of Comparative Zoology 146 (9): 411-472.

Rosa, A.O. 2000. Zooarqueologia de alguns sítios arqueológicos do pantanal sul-matogrossense. Clio, Série Arqueológica, 14: 327-342.

Schaefer, S.A. 1987. Osteology of Hypostomus plecostomus (Linnaeus), with a phylogenetic analysis of the Loricariid 
subfamilies (Pisces: Siluroidei). Contributions in Science, Natural History Museum of Los Angeles County 394: 131.

VARI, R.P. 1983. Phylogenetic relationships of the families Curimatidae, Prochilodontidae, Anostomidae, and Chilodontidae (Pisces: Characifromes). Washington, Smithsonian Contributions to Zoology 378, 60p.
Weitzman, S.H. 1962. The osteology of Brycon meeki, a generalized Characid fish, with na osteological definition of the family. Stanford Ichthyological Bulletin 8: 1-77.

Submitted: 23.IX.2008; Accepted: 25.VIII.2009.

Editorial responsibility: Wolmar B. Wosiacki

Apêndice 1. Lista dos esqueletos de peixes utilizados para composição da coleção de referência.

Acestrorhynchidae: Acestrorhynchus pantaneiro Menezes, 1992, UFRGS 5467, UFRGS 5468, UFRGS 5469, UFRGS 5470. Achiridae: Catathyridium garmani (Jordan 1889), UFRGS 5486. Anostomidae: Leporinus obtusidens (Valenciennes, 1847), UFRGS 5490. Callichthyidae: Hoplosternum litoralis Hancock, 1828, UFRGS 5479. Characidae: Brycon orbignyanus (Valenciennes 1850), UFRGS 5385, UFRGS 5386, UFRGS 5387, UFRGS 5388, UFRGS 5389; Galeocharax humerallis (Valenciennes, 1834), UFRGS 5473; Oligosarcus jenynsii (Gunther, 1864), UFRGS 5471, UFRGS 5472; Salminus brasiliensis, UFRGS 5489. Cichlidae: Crenicichla lepidota Heckel, 1840, UFRGS 5410; UFRGS 5447, UFRGS 5448, UFRGS 5449, UFRGS 5450, UFRGS 5451, UFRGS 5452, UFRGS 5453; Crenicichla sp. UFRGS 5454, UFRGS 5455, UFRGS 5456, UFRGS 5457; Crenicichla vittata Heckel, 1840, UFRGS 5383, UFRGS 5384, UFRGS 5474, UFRGS 5475; Geophagus brasiliensis (Quoy \& Gaimard, 1824), UFRGS 5412, UFRGS 5488; Gymnogeophagus sp., UFRGS 5499. Curimatidae: Cyphocharax voga (Hensel, 1870), UFRGS 5408, UFRGS 5409. Erythrinidae: Hoplias malabaricus (Bloch, 1794), UFRGS 5369; UFRGS 5370, UFRGS 5371, UFRGS 5372, UFRGS 5373, UFRGS 5374, UFRGS 5375, UFRGS 5376, UFRGS 5377, UFRGS 5378, UFRGS 5379, UFRGS 5381, UFRGS 5382,. UFRGS 5380; UFRGS 5458, UFRGS 5459, UFRGS 5460, UFRGS 5461, UFRGS 5462, UFRGS 5463, UFRGS 5464, UFRGS 5491, UFRGS 5493; UFRGS 5492. Heptapteridae: Rhamdia sp., UFRGS 5476, UFRGS 5477, UFRGS 5478 ; UFRGS 5506. Loricariidae: Hemiancistrus fuliginosus Cardoso \& Malabarba, 1999, UFRGS 5404; Hypostomus commersoni Valenciennes, 1836, UFRGS 5482; Hypostomus isbrueckeri Reis, Weber \& Malabarba, 1990, UFRGS 5393, UFRGS 5394, UFRGS 5395, UFRGS 5396, UFRGS 5397, UFRGS 5398, UFRGS 5399, UFRGS 5400, UFRGS 5401, UFRGS 5402, UFRGS 5403; Hypostomus regani (Ihering, 1905), UFRGS 5481; Pogonopoma obscurum Quevedo \& Reis, 2002. UFRGS 5391, UFRGS 5390. Pimelodidae: Megalonema platana (Gunther, 1880), UFRGS 5480; Pimelodus maculatus Lacépède, 1803, UFRGS 5483, UFRGS 5484, UFRGS 5485; Steindachneridion scripta MirandaRibeiro, 1918, UFRGS 5392, UFRGS 5411. Prochilodontidae: Prochilodus lineatus (Valenciennes, 1836), UFRGS 5405, UFRGS 5507 Sciaenidae: Pachyurus bonariensis Steindachner, 1879, UFRGS 5465, UFRGS 5466. Synbranchidae: Synbranchus marmoratus (Bloch, 1795), UFRGS 5406, UFRGS 5407, UFRGS 5487.

Apêndice 2. Espinhos peitorais de siliriformes utilizados para composição da coleção de referência utilizada no estudo.

Auchenipteridae: Auchenipterus nigripinnis (Boulenger, 1895), MCP 20747 (1:4); Auchenipterus osteomystax (Miranda-Ribeiro, 1918), MCP 13101 (1:32); Trachelyopterus albicrux (Berg, 1901), MCP 5707 (1:4); Trachelyopterus teaguei (Devincenzi, 1942), MCP 16794 (1:2). Loricariidae: Ancistrus taunayi Miranda-Ribeiro, 1918, MCP 23139 (1);

Loricariidae: Hemiancistrus fuliginosus, MCP 18539 (1); Hypostomus aspilogaster (Cope, 1894), MCP s/n; Hypostomus commersoni, MCP 6630 (1:5), MCP 10489 (1:6), MCP 18947 (1:8); Hypostomus isbrueckeri, MCP 18530 (1:14); Hypostomus luteus (Godoy, 1980), MCP 12735 (1:2); Hypostomus regani, MCP 11860 (1:3); Hypostomus roseopunctatus Reis, Weber \& Malabarba, 1990, MCP 13138 (1:4); Hypostomus uruguayensis Reis, Weber e Malabarba, 1990, MCP 18953 (1:4); Loricariichthys platymetopon Isbrücker \& Nijssen 1979, MCP 10338 (1:3).Pimelodidae: Iheringichthys labrosus (Lütken 1874), MCP 11841 (1:2); Megalonema platana, MCP 13224. (1:1); Parapimelodus valenciennis (Lütken, 1874), MCP 12431 (1:3); Pimelodus absconditus Azpelicueta, 1995, MCP 13142 (1:2); Pimelodus sp., MCP 20740 (1:2); Steindachneridion scripta, MCP 18443 (1:1). Heptapteridae: Rhamdia sp. MCP 18885 (1:2).

Apêndice 3. Lista dos espécimes medidos para composição das curvas de regressão.

Pogonopoma obscurum (162-240 mm CP; 166-500g): MCP 12329 (4); MCP 12302 (3), MCP 12303 (1), MCP 22029 (2), UFSC 7F4030MC (9), UFSC 7F 3040 MC (3), UFSC 7F5030 MC (2), UFSC 4R1 F4030 PX (4), UFSC 12M4R1 M3030 PMR (1), UFSC 4R1 3040 PX (7). Hemiancistrus fuliginosus (86-155 mm CP; 26-110 g): MCP 8720 (1), MCP 18512 (4:15), MCP 18539 (4), UFSC 2MF 5020 FO (12), UFSC 4R1 M2520 PX (3), UFSC 4R1 M2020 PX (1), UFSC 2MF M 2020 (15), UFSC 2MF 5020 FO (6).

Hypostomus isbrueckeri (92-135 mm CP; 15,2-238 g): MCP 19377 (2), MCP 13395 (3:5), UFSC 7F 3040 MC (1), UFSC 12M4R1 M3030 PMR (1), UFSC 4R1 M2520 PX (4), UFSC 4R1 M3030 PX (1), UFSC 12F 3040 MF (4), UFSC 12M 4R1 M3030 LML (1), UFSC 4R1 F5030 PX (3), UFSC 12F 3040 MPB (1), UFSC 4R1 3040 PX 7 (13), UFSC 12M 4R1 M3015 MPT (1), UFSC $12 F 3040$ MPB (1), UFSC 
2MF M2020 (1). H. luteus (100-250 mm CP; 30-370 g): MCP 13137 (6), MCP 12806 (2), MCP 12807 (2), MCP 12809 (7), MCP 12493 (1), MCP 13000 (1). H. roseopunctatus (98-230 mm CP; 50-330 g): MCP 18581 (1), MCP 132602 espécimes, MCP 19016 (2), MCP 13138 (4), MCP 18434 (1), MCP 12442 (1), MCP 18454 (1), MCP 19336 (1), MCP 18947 (4:8), UFSC 12 F 3040 MPB (3), UFSC F 3020 I (1). H. regani (110-290 mm CP; 50-600 g): MCP 28609 (3), MCP 11839 (1), MCP 19989 (1:3), MCP 11726 (1), MCP 13208 (1:2), МCP 11772 (1), MCP 5756 (1), МCP 12658 (1), МCP 18513 (1), MCP 18954 (1), MCP 10470 (2), MCP 117811 espécime, MCP 20737 (7). H. uruguayensis (132-230 mm CP; 50-250 g): MCP 16263 (2), MCP 13419 (1), MCP 20736 (2), MCP 13256 (1), МCP 13284 (1), МCP 18953 (2:4), МCP 13824 (2), МCP 19990 (3), MCP 28911 (1), MCP 18955 (1:2). H. aspilogaster (84-270 mm CP; 28-350 g): MCP 18955 (1), MCP 87090 (1), MCP 12141 (7:9), MCP 14279 (1), MCP 28085 (1), MCP 10884 (1), MCP 23762 (1), MCP 213195 espécimes, MCP 173691 espécime, MCP 11265 (2), MCP 10886 (1), MCP 8995 (2), MCP 9696 (1:9), MCP 11258 (2), MCP 10877 (1), MCP 18767 (2). H. commersoni (160-440 mm CP; 124-1426 g): UFSC 12 F 5030 MF (2), UFSC 4R1 M3030 PX (1), UFRGS (23).

Crenicichla vittata (123-245 mm CP; 16-240 g): MCP 28610 (1), MCP 15803 (1), MCP 14650 (5), MCP 211281 espécime, MCP 12646 (1), MCP 13538 (1), MCP 11894 (2), MCP 13228 (1:3), MCP 18932 (1), MCP 22034 (1), MCP 26810 (1). C. lepidota (90-180 mm CP; 20-195 g): МCP 1985 (2:3), МCP 17551 (1), МCP 6917 (2), МСP 22165 (1), MCP 13093 (2), MCP 1580 (1), MCP 18387 (5), MCP 19994 (2), MCP 15758 (1:12), MCP 15759 (1:3), MCP 16474 (1:3), MCP 10557 (2:4). C. minuano Lucena \& Kullander,1992 (92-195 mm CP; 20-110 g): МCP 19034 (2), МCP 18938 (2:6), MCP 20753 (1), MCP 16281 (1), MCP 16280 (2:4), MCP 18743 (1), MCP 22033 (4:5), MCP 28613 (1), MCP 20922 (3:9), MCP 18942 (3). C. scottii (Eigenmann, 1907) (125-180 mm CP; 50-325g MCP 10898 (3:8), MCP 11212 (6:29), MCP 14305 (1). C. gaucho Lucena \& Kullander, 1992 (76-108,7 mm CP; 15-25 g): MCP 26080 (7), МCP 12770 7:96). C. igara Lucena \& Kullander, 1992 (107-230 mm CP; 20-200 g): MCP 18705 (1), MCP 20862 (1), MCP 18713 (1), MCP 12259 (1), MCP 12257 (5), MCP 18927 (4). C. missioneira Lucena \& Kullander, 1992 (93-195 mm CP; $10-140$ g): MCP 19054 (1), MCP 19753 (4:6), MCP 19754 (2), МCP 20750 (1), MCP 16282 (1:2), MCP 22824 (3), MCP 18929 (3), MCP 28611 (2). C. tendybaguassu Lucena \& Kullander, 1992 (98-150 mm CP; $30-85$ g): MCP 19030 (1), MCP 18940 (1), MCP 20920 (2:4), MCP 18934 (2), MCP 20921 (1), MCP 18715 (1). C. jurubi Lucena \& Kullander, 1992 (106-250 mm CP; 50-270 g): MCP 18708 (1), МCP 19398 (1), MCP 19937 (1), MCP 18702 (5:7). C. prenda Lucena \& Kullander, 1992 (75-94 mm CP; 35-55 g): MCP 20861 (1), MCP 20892 (1:3), MCP 20863 (3:8), MCP 20894 (1). C. celidochilus Casciotta, 1987 (105-210 mm CP; 50-180 g): Lotes MCP 19766 (1:2), MCP 14322 (1), MCP 11881 (1), MCP 12861 (1), MCP 12864 (1), MCP 18944 (1), MCP 18876 (4), MCP 13105 (1:2), MCP 12353 (1), MCP 12861 (1), MCP 18717 (1), MCP 20771 (1:2). 